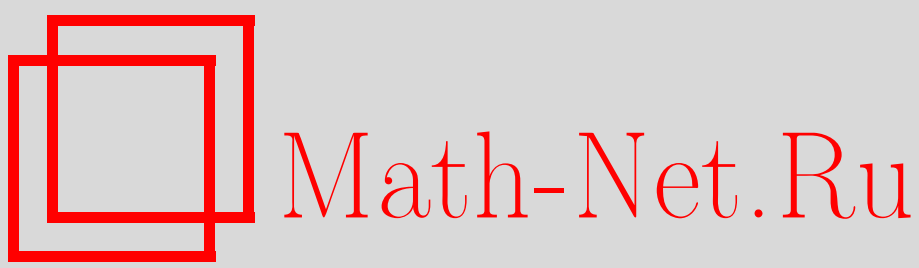

А. В. Калиниченко, М. А. Плиев, Равенство треугольника в гильбертовых $A$ модулях, Изв. вузов. Матем., 2019, номер 10, 38-45

DOI: https://doi.org/10.26907/0021-3446-2019-10-38-45

Использование Общероссийского математического портала Math-Net.Ru подразумевает, что вы прочитали и согласны с пользовательским соглашением

http://www . mathnet.ru/rus/agreement

Параметры загрузки:

IP: 54.237 .206 .68

26 апреля 2023 г., $16: 34: 27$

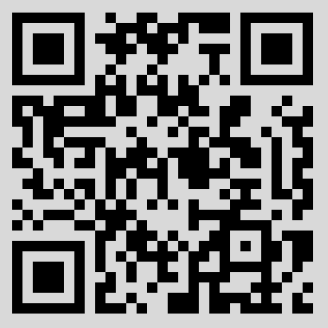


Известия вузов. Математика

2019, № 10 , c. 38-45 https://kpfu.ru/science/nauchnye-izdaniya/ivrm

e-mail: izvuz.matem@kpfu.ru

\title{
А.В.КАЛИНИЧЕНКО, М.А.ПЛИЕВ
}

\section{РАВЕНСТВО ТРЕУГОЛЬНИКА В ГИЛЬБЕРТОВЫХ $A$-МОДУЛЯХ}

\begin{abstract}
Аннотация. Установлено, что для произвольных элементов $x$ и $y$ гильбертова $A$-модуля $M$ над локальной $C^{*}$-алгеброй $A$ обобщенное равенство треугольника $|x+y|=|x|+|y|$ выполняется тогда и только тогда, когда $\langle x, y\rangle=|x||y|$.
\end{abstract}

Ключевые слова: локальная $C^{*}$-алгебра, гильбертов $A$-модуль, локальное гильбертово пространство, модульно компактный оператор, *-гомоморфизм, равенство треугольника.

УДК: 517.98: 519.46

DOI: $10.26907 / 0021-3446-2019-10-38-45$

\section{ВВЕДЕНИЕ}

Многие классические неравенства анализа, справедливые для чисел и функций, допускают обобщение для некоммутативных матриц и операторов. В последние годы операторные аналоги различных классических формул были установлены в ряде работ [1]-[3]. Стоит отметить, что прямой перенос известных формул для некоммутативных переменных часто бывает невозможен. Так обстоит дело с хорошо известным неравенством треугольника для модуля $|x+y| \leq|x|+|y|$, справедливым как в числовых полях $\mathbb{R}$ и $\mathbb{C}$, так и в классических функциональных пространствах непрерывных и измеримых функций $C[0,1]$ и $L_{p}[0,1]$ $(0 \leq p \leq \infty)$. Данное неравенство в общем случае нарушается уже в простейшей некоммутативной алгебре $M_{2}(\mathbb{C})$ квадратных $2 \times 2$ комплексных матриц. В качестве матриц $x$ и $y$, для которых неравенство не выполняется, следует взять

$$
x=\left(\begin{array}{ll}
1 & 0 \\
0 & 1
\end{array}\right), y=\left(\begin{array}{ll}
0 & 0 \\
1 & 0
\end{array}\right) .
$$

Подробности можно найти в [4]. Напомним, что $|x|=\left(x^{*} x\right)^{1 / 2}$ для любой матрицы $x \in$ $M_{n}(\mathbb{C})$. Тем не менее неравенство треугольника для любой пары $x, y \in M_{n}(\mathbb{C})$ справедливо в следующей форме:

$$
|x+y| \leq u|x| u^{*}+v|y| v^{*},
$$

где $u, v$ - некоторые унитарные матрицы, зависящие от $x$ и $y([5])$. Равенство треугольника в виде $|x+y|=|x|+|y|$ для элементов операторных алгебр и модулей изучалось в работах [6], [7].

Поступила в редакцию 29.08.2018, после доработки 29.08.2018. Принята к публикации 19.12.2018.

Благодарности. М.А.Плиев поддержан грантом Российского фонда фундаментальных исследований 17-51-12064. 
В настоящей статье продолжим данную линию исследований и найдем критерий выполнения равенства треугольника для произвольных элементов $x, y$ гильбертова $A$-модуля $\mathcal{M}$ над локальной $C^{*}$-алгеброй $A$.

\section{1. ПРЕДВАРИТЕЛЬНЫЕ СВЕДЕНИЯ}

Цель этого раздела - зафиксировать терминологию и используемые в тексте обозначения. Стандартный источник по теории $C^{*}$-алгебр - монография [8]. Все необходимые сведения о локальных $C^{*}$-алгебрах и гильбертовых $A$-модулях над ними можно найти в [9], [10]. Все алгебры рассматриваются над полем комплексных чисел.

Всюду ниже будем полагать, что внутренние произведения сопряженно линейны по первой переменной и линейны по второй переменной. Пространство линейных, непрерывных операторов, действующих из гильбертова пространства $H$ в гильбертово пространство $K$, обозначается через $L(H, K)$ и $L(H):=L(H, H)$.

Пусть $A$ - инволютивная алгебра и $p: A \rightarrow \mathbb{R}_{+}-$полунорма на $A$, удовлетворяющая следующим условиям:

1) $p(x y) \leq p(x) p(y)$ для любых $x, y \in A$,

2) $p(x)=p\left(x^{*}\right)$ для любого $x \in A$.

Если, кроме того, для любого $x \in A$ справедливо равенство $p\left(x^{*} x\right)=p(x)^{2}$, то $p$ называется $C^{*}$-полунормой. Инволютивная топологическая алгебра, полная относительно отделимой топологии, задаваемой разделяющим, направленным семейством $C^{*}$-полунорм $\left(p_{\lambda}\right)_{\lambda \in \Lambda}$, называется локальной $C^{*}$-алгеброй. Рассмотрим некоторые примеры.

Пример 1. Каждая $C^{*}$-алгебра является локальной $C^{*}$-алгеброй.

Пример 2. Каждая замкнутая *-подалгебра локальной $C^{*}$-алгебры также является локальной $C^{*}$-алгеброй.

Пример 3. Пусть $Q$ - вполне регулярное топологическое пространство и пусть $C(Q)-$ алгебра непрерывных комплекснозначных функций, заданных на $Q$. Для каждого компактного множества $K \subset Q$ введем полунорму

$$
p_{K}(f):=\sup _{x \in K}|f(x)|, \quad f \in C(Q) .
$$

Тогда $p_{K}$ будет $C^{*}$-полунормой и топологическая алгебра $C(Q)$ с топологией, задаваемой разделяющим, направленным семейством полунорм, является локальной $C^{*}$-алгеброй.

Пример 4. Пусть $\Lambda$ - частично упорядоченное, направленное вверх индексное множество, $\left\{H_{\lambda}\right\}_{\lambda \in \Lambda}-$ семейство гильбертовых пространств таких, что $H_{\lambda} \subset H_{\mu}$ и

$$
\langle\cdot, \cdot\rangle_{\lambda}=\left.\langle\cdot, \cdot\rangle_{\mu}\right|_{H_{\lambda}}, \quad \lambda \leq \mu,
$$

где $\langle\cdot, \cdot\rangle_{\lambda}$ - скалярное произведение в пространстве $H_{\lambda}, \lambda \in \Lambda$. Рассмотрим локально выпуклое пространство

$$
\mathcal{H}:=\lim _{\rightarrow} H_{\lambda}=\bigcup_{\lambda} H_{\lambda} .
$$

Это пространство с топологией индуктивного предела называется локалъным гилъбертовым пространством. Линейный оператор $T: \mathcal{H} \rightarrow \mathcal{H}$ называется непрерывным, если имеет место представление

$$
T=\lim _{\rightarrow} T_{\lambda}, \quad T_{\lambda} \in L\left(H_{\lambda}\right),
$$

где $\left(T_{\lambda}\right)_{\lambda \in \Lambda}-$ семейство операторов $T_{\lambda} \in L\left(H_{\lambda}\right)$ для любого $\lambda \in \Lambda$. Векторное пространство всех линейных непрерывных операторов на $\mathcal{H}$ обозначается через $L(\mathcal{H})$. Отметим, что 
$L(\mathcal{H})$ является также и алгеброй, и с каждым семейством $\left(T_{\lambda}\right)_{\lambda \in \Lambda}$ можно связать семейство $\left(T_{\lambda}\right)_{\lambda \in \Lambda}^{*}$, где $T_{\lambda}^{*} \in L\left(H_{\lambda}\right)$. Тогда отображение

$$
*: L(\mathcal{H}) \rightarrow L(\mathcal{H}), \quad T \mapsto T^{*}=\lim _{\rightarrow} T_{\lambda}^{*}
$$

будет инволюцией, заданной на $L(\mathcal{H})$. Если $\|\cdot\|_{\lambda}$ - операторная норма, заданная на $L\left(H_{\lambda}\right)$, то функция

$$
p_{\lambda}(T):=\left\|T_{\lambda}\right\|_{\lambda}, \quad T \in L(\mathcal{H}),
$$

будет $C^{*}$-полунормой на $L(\mathcal{H})$ для каждого $\lambda \in \Lambda$ и $L(\mathcal{H})-$ это локальная $C^{*}$-алгебра относительно топологии, задаваемой разделяющим семейством полунорм $\left\{p_{\lambda}\right\}_{\lambda \in \Lambda}$.

Пусть $A$ - локальная $C^{*}$-алгебра относительно топологии, задаваемой разделяющим семейством полунорм $\left\{p_{\lambda}\right\}_{\lambda \in \Lambda}$. Предгилъбертовым $A$-модулем называется комплексное векторное пространство $\mathcal{M}$, являющееся также правым $A$-модулем, снабженное $A$-значным внутренним произведением, т. е. отображением $\langle\cdot, \cdot\rangle: \mathcal{M} \times \mathcal{M} \rightarrow A$ со свойствами

$$
\begin{gathered}
\langle x, \alpha y+\beta z\rangle=\langle x, y\rangle \alpha+\langle x, z\rangle \beta \text { для любых } x, y, z \in \mathcal{M} ; \quad \alpha, \beta \in \mathbb{C} ; \\
\langle x, y a\rangle=\langle x, y\rangle a \text { для любых } x, y \in \mathcal{M} ; \quad a \in A ; \\
\langle x, y\rangle^{*}=\langle y, x\rangle \text { для любых } x, y \in \mathcal{M} ; \\
\langle x, x\rangle \geq 0 \text { для любого } x \in \mathcal{M} ; \\
\langle x, x\rangle=0 \Leftrightarrow x=0 \text { для любого } x \in \mathcal{M} .
\end{gathered}
$$

Будем говорить, что $\mathcal{M}$ - гилъбертов $A$-модуль, если $\mathcal{M}$ является полным топологическим векторным пространством относительно топологии, задаваемой разделяющим, направленным семейством полунорм

$$
\bar{p}_{\lambda}(x):=\sqrt{p_{\lambda}(\langle x, x\rangle)}, \quad x \in \mathcal{M}, \lambda \in \Lambda .
$$

Заметим, что локальная $C^{*}$-алгебра $A$ является частным случаем гильбертова $A$-модуля. Здесь $\mathcal{M}=A$, а внутреннее произведение имеет вид

$$
\langle x, y\rangle:=x^{*} y, \quad x, y \in A .
$$

Пусть $A$ - локальная $C^{*}$-алгебра и $\mathcal{M}, \mathcal{N}$ - гильбертовы $A$-модули над $A$. Для элемента $x \in \mathcal{M}$ положим $|x|:=\langle x, x\rangle^{1 / 2}$. В случае, когда $A$ совпадает с $\mathcal{M}$, получаем традиционное определение модуля $|x|=\left(x^{*} x\right)^{1 / 2}$. Линейный оператор $T: \mathcal{M} \rightarrow \mathcal{N}$ называется $A$-линейным, если для любых $x \in \mathcal{M}, a \in A$ справедливо равенство $T(x a)=T(x) a$. Множество всех непрерывных $A$-линейных операторов из $\mathcal{M}$ в $\mathcal{N}$ обозначается $L_{A}(\mathcal{M}, \mathcal{N})$ или просто $L(\mathcal{M}, \mathcal{N})$, если ясно, о какой алгебре $A$ идет речь. Говорят, что линейный оператор $T: \mathcal{M} \rightarrow \mathcal{N}$ допускает сопряжсенный, если существует линейный оператор $S: \mathcal{N} \rightarrow \mathcal{M}$ такой, что $\langle T x, y\rangle=\langle x, S y\rangle$ для любых элементов $x \in \mathcal{M}, y \in \mathcal{N}$. При этом $S$ называется сопряженным оператором к $T$ и обозначается $T^{*}$. Векторное пространство всех линейных операторов $T: \mathcal{M} \rightarrow \mathcal{N}$, допускающих сопряженный, обозначается через $\mathcal{L}_{A}(\mathcal{M}, \mathcal{N})$. Известно ([10], теорема 2.2.6), что каждый линейный оператор, допускающий сопряжение, является $A$-линейным и $\mathcal{L}_{A}(\mathcal{M}):=\mathcal{L}_{A}(\mathcal{M}, \mathcal{M})$ является локальной $C^{*}$-алгеброй. Для $x \in \mathcal{N}$, $y \in \mathcal{M}$ положим

$$
\theta_{x, y}(z):=x\langle y, z\rangle, \quad z \in \mathcal{M}
$$

Ясно, что для любых $x \in \mathcal{N}, y \in \mathcal{M}$ отображение $\theta_{x, y}$ является $A$-линейным оператором из $\mathcal{M}$ в $\mathcal{N}$. Известно $\left([10]\right.$, с. 38), что $\theta_{x, y}^{*}=\theta_{y, x}$ и для любых $x \in \mathcal{N}, y \in \mathcal{M}$ справедливо включение $\theta_{x, y} \in \mathcal{L}(\mathcal{M}, \mathcal{N})$. Линейное подпространство в $\mathcal{L}_{A}(\mathcal{M}, \mathcal{N})$, порожденное элементами $\theta_{x, y}, x \in \mathcal{N}, y \in \mathcal{M}$, обозначается $\Theta_{A}(\mathcal{M}, \mathcal{N})$ или просто $\Theta(\mathcal{M}, \mathcal{N})$. Замыкание $\Theta_{A}(\mathcal{M}, \mathcal{N})$ в 
$\mathcal{L}_{A}(\mathcal{M}, \mathcal{N})$ обозначается через $\mathcal{K}_{A}(\mathcal{M}, \mathcal{N})$ или просто через $\mathcal{K}(\mathcal{M}, \mathcal{N})$. Элементы $\mathcal{K}(\mathcal{M}, \mathcal{N})$ называются модульно компактными операторами из $\mathcal{M}$ в $\mathcal{N}$. Известно ([10], замечание 2.3.3), что $\mathcal{K}(\mathcal{M}):=\mathcal{K}(\mathcal{M}, \mathcal{M})$ является локальной $C^{*}$-алгеброй и двусторонним $*$-идеалом в $\mathcal{L}(\mathcal{M})$.

Теория гильбертовых $A$-модулей тесно связана с многими разделами современной математики ([10]). Операторы, действующие в $A$-модулях, изучались в работах [11]-[14].

\section{2. ОСНОВНОЙ РЕЗУЛЬТАТ}

Установим основной результат статьи - критерий равенства $|x+y|=|x|+|y|$, где $x$ и $y$ - элементы гильбертова $A$-модуля над локальной $C^{*}$-алгеброй.

Предварительно потребуется несколько вспомогательных утверждений.

Пусть $H$ и $K$ - гильбертовы пространства. Напомним, что линейный непрерывный опеpaтор $T: H \rightarrow K$ называется частичной изометрией, если он изометричен на замкнутом подпространстве $\operatorname{ker}(T)^{\perp}$, где $\operatorname{ker}(T):=\{v \in H: T v=0\}$ и $\operatorname{ker}(T)^{\perp}-$ ортогональное дополнение подпространства $\operatorname{ker}(T)$. Отметим также, что операторы $T^{*} T$ и $T T^{*}$ являются ортогональными проекторами на пространства $\operatorname{ker}(T)^{\perp}$ и $\operatorname{Im}(T):=\{T v: v \in H\}$ соответственно.

Лемма 2.1 ([6], теорема 2.3). Пусть $H$ - гилъбертово пространство, $T, S \in L(H)$. Тогда следующие условия эквивалентны:

a) $|T+S|=|T|+|S|$

b) существует частичная изометрия $U \in L(H)$ такая, что $T=U|T| u S=U|S|$.

Отметим также, что вышеприведенные представления: $T=U|T|$ и $S=U|S|$ для операторов $T$ и $S$ соответственно, называются полярными разложениями операторов $T$ и $S$. При этом имеют место равенства ([8], теорема 2.3.4)

$$
U^{*} U|T|=|T| ; U^{*} U|S|=|S| .
$$

Пусть $\Lambda$ - направленное вверх множество, $\left\{H_{\lambda}\right\}_{\lambda \in \Lambda}-$ семейство гильбертовых пространств и $\mathcal{H}=\bigcup_{\lambda \in \Lambda} H_{\lambda}-$ локальное гильбертово пространство. Оператор $T \in L(\mathcal{H})$ называется частичной изометрией, если для любого $\lambda \in \Lambda$ частичными изометриями являются операторы $T_{\lambda}: H_{\lambda} \rightarrow H_{\lambda}$. Ниже покажем, что результаты леммы 2.1 остаются справедливыми и для непрерывных операторов в локальных гильбертовых пространствах.

Лемма 2.2. Пусть $\Lambda$ - направленное вверх множество, $\left\{H_{\lambda}\right\}_{\lambda \in \Lambda}-$ семейство гильбертовых пространств и $\mathcal{H}=\bigcup_{\lambda \in \Lambda} H_{\lambda}-$ локальное гилъбертово пространство, $T, S \in L(\mathcal{H})$. Тогда следующие условия эквивалентны:

a) $|T+S|=|T|+|S|$;

b) существует частичная изометрия $U \in L(\mathcal{H})$ такая, что $T=U|T|$ u $S=U|S|$.

Доказательство. a) $\Rightarrow$ b). Возьмем произвольный оператор $R \in L(\mathcal{H})$. По определению

$$
|R|=\left(R^{*} R\right)^{1 / 2} ; \quad|R|_{\lambda}=\left(R_{\lambda}^{*} R_{\lambda}\right)^{1 / 2}=\left|R_{\lambda}\right| \in L\left(H_{\lambda}\right), \quad \lambda \in \Lambda .
$$

Пусть для операторов $T, S \in L(\mathcal{H})$ выполняется равенство треугольника. Тогда

$$
\left|(T+S)_{\lambda}\right|=|T+S|_{\lambda}=|T|_{\lambda}+|S|_{\lambda}=\left|T_{\lambda}\right|+\left|S_{\lambda}\right| .
$$

Согласно лемме 2.1 для каждого $\lambda \in \Lambda$ найдется частичная изометрия $U_{\lambda} \in L\left(H_{\lambda}\right)$ такая, что $T_{\lambda}=U_{\lambda}\left|T_{\lambda}\right|$ и $S_{\lambda}=U_{\lambda}\left|S_{\lambda}\right|$. Положим

$$
U:=\lim _{\rightarrow} U_{\lambda}, U_{\lambda} \in L\left(H_{\lambda}\right) .
$$


Получаем, что оператор $U \in L(\mathcal{H})$ является искомой частичной изометрией.

b) $\Rightarrow$ a). Справедлива следующая цепочка равенств:

$$
T_{\lambda}=U_{\lambda}|T|_{\lambda}=U_{\lambda}\left|T_{\lambda}\right|, \quad S_{\lambda}=U_{\lambda}|S|_{\lambda}=U_{\lambda}\left|S_{\lambda}\right| .
$$

Снова применяя лемму 2.1, имеем

$$
|T+S|_{\lambda}=\left|T_{\lambda}+S_{\lambda}\right|=\left|T_{\lambda}\right|+\left|S_{\lambda}\right|=|T|_{\lambda}+|S|_{\lambda}, \lambda \in \Lambda .
$$

Таким образом, получили $|T+S|=|T|+|S|$.

Заметим также, что в условиях леммы 2.2 для частичной изометрии $U: L(\mathcal{H}) \rightarrow L(\mathcal{H})$ имеет место полезный факт

$$
U_{\lambda}^{*} U_{\lambda}\left|T_{\lambda}\right|=\left|T_{\lambda}\right|, \quad U_{\lambda}^{*} U_{\lambda}\left|S_{\lambda}\right|=\left|S_{\lambda}\right|, \quad \lambda \in \Lambda \Rightarrow U^{*} U|T|=|T|, \quad U^{*} U|S|=|S| .
$$

Для локальных $C^{*}$-алгебр справедлив аналог хорошо известной для $C^{*}$-алгебр $Г$ ГС-конструкции. Напомним, что линейное отображение $\varphi: A \rightarrow B$ локальных $C^{*}$-алгебр $A$ и $B$ называется $*$-гомоморфизмом, если для любых $a, b \in A$ выполняются равенства

$$
\varphi(a b)=\varphi(a) \varphi(b), \varphi\left(a^{*}\right)=\varphi(a)^{*} .
$$

Представлением локальной $C^{*}$-алгебры $A$ называется пара $(\mathcal{H}, \varphi)$, где $\mathcal{H}$ - локальное гильбертово пространство и $\varphi: A \rightarrow L(\mathcal{H})-$ непрерывный $*$-гомоморфизм. Представление называется точным, если $\varphi$ инъективен. Полезно также отметить следующее свойство произвольного $*$-гомоморфизма $\varphi: A \rightarrow B$

$$
|\varphi(a)|=\varphi(|a|), \quad a \in A .
$$

Действительно, имеет место цепочка равенств

$$
|\varphi(a)|^{2}=\varphi(a)^{*} \varphi(a)=\varphi\left(a^{*} a\right)=\varphi\left(|a|^{2}\right)=(\varphi(|a|))^{2} .
$$

Отсюда, используя единственность квадратного корня положительного элемента локальной $C^{*}$-алгебры ([9], предложение 10.13), выводим требуемое.

Лемма 2.3 ([15], теорема 5.1). Каждая локальная $C^{*}$-алгебра допускает точное представление.

Сейчас готовы доказать необходимые и достаточные условия равенства треугольника для элементов локальной $C^{*}$-алгебры.

Лемма 2.4. Пусть $\mathcal{A}$ - локальная $C^{*}$-алгебра и $a, b \in \mathcal{A}$. Тогда следующие условия эквивалентны:

(1) $|a+b|=|a|+|b|$,

(2) $a^{*} b=|a||b|$.

Доказательство. $(2) \Rightarrow(1)$. Предположим, что $|a+b|=|a|+|b|$. Согласно лемме 2.3 найдется локальное гильбертово пространство $\mathcal{H}$ и инъективный непрерывный $*$-гомоморфизм $\varphi: A \rightarrow L(\mathcal{H})$. Тогда $\varphi(a), \varphi(b) \in L(\mathcal{H})$ и имеют место равенства

$$
|\varphi(a)+\varphi(b)|=|\varphi(a+b)|=\varphi(|a+b|)=\varphi(|a|+|b|)=\varphi(|a|)+\varphi(|b|) .
$$

Применяя лемму 2.2 , найдем частичную изометрию $U \in L(\mathcal{H})$ такую, что

$$
\varphi(a)=U|\varphi(a)|, \quad \varphi(b)=U|\varphi(b)|, \quad U^{*} U|\varphi(a)|=|\varphi(a)|, \quad U^{*} U|\varphi(b)|=|\varphi(b)| .
$$

Далее имеем

$$
\varphi\left(a^{*} b\right)=\varphi(a)^{*} \varphi(b)=(U|\varphi(a)|)^{*} U|\varphi(b)|=|\varphi(a)| U^{*} U|\varphi(b)|=|\varphi(a)||\varphi(b)|=\varphi(|a|) \varphi(|b|)=\varphi(|a||b|) .
$$

В силу инъективности непрерывного *-гомоморфизма выводим, что $a^{*} b=|a||b|$. 
$(1) \Rightarrow(2)$. Предположим, что $a^{*} b=|a||b|$. Тогда $b^{*} a=\left(a^{*} b\right)^{*}=|b||a|$ и справедливы следующие равенства:

$$
|a+b|^{2}=(a+b)^{*}(a+b)=a^{*} a+a^{*} b+b^{*} a+b^{*} b=|a|^{2}+\left.|a| b|+| b|| a|+| b\right|^{2}=(|a|+|b|)^{2} .
$$

Отсюда в силу единственности квадратного корня положительного элемента локальной $C^{*}$ алгебры ([15], предложение 2.1) выводим $|a+b|=|a|+|b|$.

Ключевым инструментом для доказательства основного результата является следующая конструкция. Пусть $A$ - локальная $C^{*}$-алгебра и $\mathcal{M}$ - гильбертов $A$-модуль. Прямая сумма $A \oplus \mathcal{M}$ также является гильбертовым $A$-модулем, где действие $A$ на $A \oplus \mathcal{M}$ и $A$-значное внутреннее произведение на $A \oplus \mathcal{M}$ задаются формулами

$$
(a, x) b=(a b, x b), \quad\langle(a, x),(b, y)\rangle=a^{*} b+\langle x, y\rangle \in A, \quad a, b \in A, \quad x, y \in \mathcal{M} .
$$

Пусть $a \in A, x, y \in \mathcal{M}$ и $T \in \mathcal{K}_{A}(\mathcal{M})$. Рассмотрим отображение $L_{a, x, y, T}: A \oplus \mathcal{M} \rightarrow A \oplus \mathcal{M}$, заданное правилом

$$
L_{a, x, y, T}(b, z)=(a b+\langle x, z\rangle, y b+T z) .
$$

Данные отображения допускают следующее описание.

Лемма 2.5 ([16]). Пусть $a \in A, x, y \in \mathcal{M} u T \in \mathcal{K}_{A}(\mathcal{M})$. Тогда $L_{a, x, y, T} \in \mathcal{L}(A \oplus \mathcal{M}) u$ $L_{a, x, y, T}^{*}=L_{a^{*}, y, x, T^{*}}$. Более того, локалъная $C^{*}$-алгебра, порожденная элементами

$$
\left\{L_{a, x, y, T}: a \in A, \quad x, y \in \mathcal{M}, \quad T \in \mathcal{K}_{A}(\mathcal{M})\right\},
$$

совпадает с $\mathcal{K}_{A}(A \oplus \mathcal{M})$.

Операторы $L_{a, x, y, T}$ удобно представлять в матричном виде

$$
L_{a, x, y, T}=\left(\begin{array}{cc}
a & x \\
y & T
\end{array}\right), \quad L_{a, x, y, T}^{*}=\left(\begin{array}{cc}
a^{*} & y \\
x & T^{*}
\end{array}\right) .
$$

Основным результатом статьи является

Теорема 2.1. Пусть $\mathcal{A}-$ локальнал $C^{*}$-алгебра, $\mathcal{M}$ - гилъбертов $A$-модуль, $x, y \in \mathcal{M}$. Тогда следующие условия эквивалентны:

(1) $|x+y|=|x|+|y|$,

(2) $\langle x, y\rangle=|x||y|$.

Доказательство. (2) $\Rightarrow(1)$. Имеют место равенства

$$
\begin{gathered}
\langle x, y\rangle=|x||y| \Rightarrow\langle y, x\rangle=\langle x, y\rangle^{*}=(|x||y|)^{*}=|y||x|, \\
|x+y|^{2}=\langle x+y, x+y\rangle=\langle x, x\rangle+\langle x, y\rangle+\langle y, x\rangle+\langle y, y\rangle=|x|^{2}+|x||y|+|y||x|+|y|^{2}=(|x|+|y|)^{2} .
\end{gathered}
$$

$(1) \Rightarrow(2)$. Отображение $a \mapsto G_{a}$, где

$$
G_{a}=\left(\begin{array}{cc}
a & 0 \\
0 & 0
\end{array}\right)
$$

является инъективным *-гомоморфизмом локальных $C^{*}$-алгебр $A$ и $\mathcal{K}_{A}(A \oplus \mathcal{M})$. $\mathrm{B}$ силу равенства $|x+y|=|x|+|y|$ выводим $G_{|x+y|}=G_{|x|}+G_{|y|}$. Далее можем написать

$$
\left(\begin{array}{cc}
|x+y| & 0 \\
0 & 0
\end{array}\right)=\left(\begin{array}{cc}
|x| & 0 \\
0 & 0
\end{array}\right)+\left(\begin{array}{cc}
|y| & 0 \\
0 & 0
\end{array}\right) .
$$

Отметим, что $G_{|x|}$ является положительным элементом алгебры $\mathcal{K}_{A}(A \oplus \mathcal{M})$ для любого $x \in \mathcal{M}$. Кроме того, для любых $a \in A, x \in \mathcal{M}$ имеем

$$
G_{|x|}^{2}(a)=|x|(|x| a)=\langle x, x\rangle a=\langle x, x a\rangle=l_{x}\left(r_{x}(a)\right)=r_{x}^{*} r_{x}(a),
$$


где через $r(x)$ и $l(x)$ обозначаются матрицы

$$
\left(\begin{array}{ll}
0 & 0 \\
x & 0
\end{array}\right) \text { и }\left(\begin{array}{ll}
0 & x \\
0 & 0
\end{array}\right)
$$

соответственно. Операторы $r(x)$ и $l(x)$ действуют на элементы модуля $A \oplus \mathcal{M}$ следующим образом:

$$
\left(\begin{array}{ll}
0 & 0 \\
x & 0
\end{array}\right)\left(\begin{array}{l}
b \\
z
\end{array}\right)=\left(\begin{array}{c}
0 \\
x b
\end{array}\right),\left(\begin{array}{ll}
0 & x \\
0 & 0
\end{array}\right)\left(\begin{array}{l}
b \\
z
\end{array}\right)=\left(\begin{array}{c}
\langle x, z\rangle \\
0
\end{array}\right), b \in A, x, z \in \mathcal{M}
$$

Ясно, что $l_{x}^{*}=r(x)$ для любого $x \in \mathcal{M}$. Тогда

$$
\left(\begin{array}{cc}
|x| & 0 \\
0 & 0
\end{array}\right)=\left|\left(\begin{array}{ll}
0 & 0 \\
x & 0
\end{array}\right)\right|
$$

Заметим, что условие (1) можно переписать в форме

$$
\left|\left(\begin{array}{cc}
0 & 0 \\
x+y & 0
\end{array}\right)\right|=\left|\left(\begin{array}{ll}
0 & 0 \\
x & 0
\end{array}\right)\right|+\left|\left(\begin{array}{ll}
0 & 0 \\
y & 0
\end{array}\right)\right| .
$$

Так как матрицы $r(|x+y|), r(|x|)$ и $r(|y|)$ являются элементами локальной $C^{*}$-алгебры $\mathcal{K}_{A}(A \oplus \mathcal{M})$, то к ним применима лемма 2.4. Отсюда выводим

$$
\left(\begin{array}{ll}
0 & 0 \\
x & 0
\end{array}\right)^{*}\left(\begin{array}{ll}
0 & 0 \\
y & 0
\end{array}\right)=\left|\left(\begin{array}{ll}
0 & 0 \\
x & 0
\end{array}\right)\right|\left|\left(\begin{array}{ll}
0 & 0 \\
y & 0
\end{array}\right)\right|
$$

Тогда

$$
\left(\begin{array}{ll}
0 & x \\
0 & 0
\end{array}\right)\left(\begin{array}{ll}
0 & 0 \\
y & 0
\end{array}\right)=\left(\begin{array}{cc}
|x| & 0 \\
0 & 0
\end{array}\right)\left(\begin{array}{cc}
|y| & 0 \\
0 & 0
\end{array}\right) .
$$

Последнее равенство эквивалентно тождеству матричных операторов

$$
\left(\begin{array}{cc}
\langle x, y\rangle & 0 \\
0 & 0
\end{array}\right)=\left(\begin{array}{cc}
|x||y| & 0 \\
0 & 0
\end{array}\right)
$$

Таким образом, $G_{\langle x, y\rangle}=G_{|x| y \mid}$. Принимая во внимание инъективность отображения $a \mapsto G_{a}$, окончательно получаем $\langle x, y\rangle=|x||y|$.

Авторы выражают искреннюю благодарность рецензенту за внимательное чтение текста и ценные замечания.

\section{ЛитеРАтУРА}

[1] Bakherad M., Moslehian M.S. Reversees and variations of Heinz inequality, Linear and Multilinear Algebra 63 (10), 1972-1980 (2015).

[2] Dadkhah A., Moslehian M.S. Gruss type inequalities for positive linear maps in $C^{*}$-algebras, Linear and Multilinear Algebra 65 (7), 1386-1401 (2017).

[3] Li X., Wu W. Operator's Jensen inequality on $C^{*}$-algebras, Acta Math. Sinica 30 (1), 35-50 (2014).

[4] Hart R. The triangle inequality in $C^{*}$-algebras, Filomat 20, 51-53 (2006).

[5] Thompson R.C. Convex and concave functions of singular values of matrix sums, Pacific J. Math. 82, 279-280 (1979).

[6] Ando T., Hayashi A. A characterization of the operator-valued triangle equality, J. Operator Theory 58 (2), 463-468 (2007).

[7] Arambasic L., Rajic R. On the $C^{*}$-valued triangle equality and inequality in Hilbert $C^{*}$-modules, Acta Math. Hungar 119 (4), 373-380 (2008).

[8] Мерфи Д. $C^{*}$-алгебры и теория операторов (Факториал, М., 1997).

[9] Fragoulopoulou M. Topological algebras with involution (Elsevier, 2005).

[10] Joiţa M. Hilbert modules over locally $C^{*}$-algebras (Univ. Bucharest Press, 2006). 
[11] Малиев И.Н., Плиев М.А. О представлении типа Стайнспринга для операторов в гилъбертовых модуллх над локалъными $C^{*}$-алгебрами, Изв. вузов. Матем., № 12, 51-58 (2012).

[12] Плиев М.А., Цопанов И.Д. О представлении типа Стайнспринга для п-наборов вполне положителъных отображений в гилъбертовых $C^{*}$-модулях, Изв. вузов. Матем., № 11, $42-49$ (2014).

[13] Masaev H. M., Pliev M. A., Elsaev Y. V. Radon-Nikodym type theorem for a covariant completely positive maps on Hilbert $C^{*}$-modules, Int. J. Math. Anal. 9 (5), 1723-1731 (2015).

[14] Moslehian M. S., Kusraev A., Pliev M. Matrix KSGNS construction and a Radon-Nikodym type theorem, Indag. Math. 28 (5), 938-952 (2017).

[15] Inoue A. Locally $C^{*}$-algebras, Mem. Faculty Sci. Kyushu Univ. Ser. A 25, 197-235 (1971).

[16] Joiţa M. On the linking algebra of Hilbert modules and Morita equivalence of locally $C^{*}$-algebras, Survey Math. and Appl.1, 23-32 (2006).

Алла Викторовна Калиниченко

Северо-Кавказский горно-металлургический институт (государственный технологический университет), ул. Николаева, д. 44, г. Владикавказ, 362021, Россия,

e-mail : kalinichenkoalla@mail.ru

Марат Амурханович Плиев

Южный Математический институт ВНЦ РАН, ул. Маркуса, д. 22, г. Владикавказ, 362027, Россия,

e-mail : plimarat@yandex.ru

\section{A.V. Kalinichenko and M.A.Pliev}

\section{The triangle equality in Hilbert $A$-modules}

Abstract. We show that for any two elements $x, y$ of Hilbert $A$-module $M$ over local $C^{*}$-algebra $A$ the generalized triangle equality $|x+y|=|x|+|y|$ holds if and only if $\langle x, y\rangle=|x||y|$.

Keywords: local $C^{*}$-algebra, Hilbert $A$-module, local Hilbert space, module compact operator, *-homomorphism, triangle equality.

Alla Viktorovna Kalinichenko

North-Caucasian Institute of Mining and Metallurgy (State Technological University), 44 Nikolaeva str., Vladikavkaz, 362021 Russia,

e-mail : kalinichenkoalla@mail.ru

Marat Amurhanovich Pliev

Southern Mathematical Institute of the Russian Academy of Sciences, 22 Markusa str., Vladikavkaz, 362027 Russia,

e-mail:plimarat@yandex.ru 